\title{
Influence of tryptophan on auxin-synthesizing ability of surfactant producer Acinetobacter calcoaceticus IMV B-7241
}

\section{Tetiana Pirog ${ }^{1,2}$, Natalia Leonova ${ }^{2}$, Daria Piatetska ${ }^{1}$, Natalia Klymenko', Tatiana Shevchuk ${ }^{2}$}

\author{
1 - National University of Food Technologies, Kyiv, Ukraine \\ 2 - Institute of Microbiology and Virology of the National Academy of Science of Ukraine, \\ Kyiv, Ukraine
}

\section{Keywords:}

Biosynthesis

Precursor

Indolyl-acetic acid

Surfactant

Acinetobacter

calcoaceticus

IMB B-7241

\section{Article history:}

Received 2.08.2019

Received in revised

form 23.12.2019

Accepted

30.03.2020

Corresponding

author:

Tetiana Pirog

E-mail:

tapirog@nudt.edu.ua

DOI:

$10.24263 / 2304-$

974X-2020-9-1-15

\section{Abstract}

Introduction. The aim of this work is to determine the optimal tryptophan concentration and the time of its addition into the culture medium of the surfactant producer Acinetobacter calcoaceticus IMV B-7241 to achieve maximum auxin synthesis.

Materials and methods. Cultivation was carried out on a liquid nutrient mineral medium using as a substrate of ethanol and waste of biodiesel production (crude glycerol). Tryptophan was added into the medium as a $1 \%$ solution in an amount of 100,200 or 300 $\mathrm{mg} / \mathrm{L}$ at the beginning of the process or at the end of the exponential growth phase ( $48 \mathrm{~h}$ of cultivation). Phytohormones were isolated by three times extraction with organic solvents from the supernatant of the culture liquid after extraction of surfactants. The qualitative and quantitative determination of gibberellins was carried out by high performance liquid chromatography.

Results and discussion. The results show that regardless of the time of addition of tryptophan in the culture medium of the strain IMV B-7241 with crude glycerol a significant increase in the synthesis of auxins compared with those on the medium without this precursor was observed. The highest concentration of auxins was achieved by adding $300 \mathrm{mg} / \mathrm{L}$ of tryptophan into the medium with both substrates. Thus, A. calcoaceticus IMV B-7241 synthesized $1404.73,1295.04$ and $4850.98 \mu \mathrm{g} / \mathrm{L}$ of auxins on the crude glycerol medium with 100, 200 and $300 \mathrm{mg} / \mathrm{L}$ tryptophan added at the end of exponential phase respectively (and without precursor the concentration of auxins was $175,4 \mu \mathrm{g} / \mathrm{L}$ ). Increased synthesis of auxins by strain IMV B-7241 correlated with the activity of tryptophan transaminase (key enzyme of biosynthesis): under cultivation on crude glycerol without precursor it was 163 $\mathrm{nmol} \cdot \mathrm{min}^{-1} \cdot \mathrm{mg}^{-1}$ of protein, while in the presence of $300 \mathrm{mg} / \mathrm{L}$ of tryptophan added at the end of the exponential growth phase the activity was in 3.2 times higher $-526 \mathrm{nmol} \cdot \mathrm{min}^{-1} \cdot \mathrm{mg}^{-1}$ of protein. The highest concentration of auxins under cultivation on ethanol was achieved when $300 \mathrm{mg} / \mathrm{L}$ of tryptophan were added at the beginning of cultivation $-2261.66 \mu \mathrm{g} / \mathrm{L}$.

Conclusion. The result of the work established the possibility of increasing by one or two orders of magnitude of synthesized auxins in the case of addition into the culture medium of A. calcoaceticus IMV B-7241 low concentrations of the precursor of their biosynthesis. 


\section{Introduction}

Previous studies have established the ability of the producer of surfactants Acinetobacter calcoaceticus IMV B-7241 to synthesize stimulatory phytohormones - auxins, cytokinins and gibberellins [1]. Such results are the basis for the development of complex preparations with growth-stimulating and antimicrobial activities against phytopathogenic bacteria properties for use in plant production.

The following studies $[2,3]$ conducted at the greenhouses of the D.K. Zabolotny Institute of Microbiology and Virology of National Academy of Sciences of Ukraine using as test cultures of tomatoes and barley, confirmed that the supernatant of the culture broth $A$. calcoaceticus IMV B-7241 has a positive effect on plant growth and development. It was found that during the treatment of barley seeds with diluted supernatant of A. calcoaceticus IMV B-7241 culture broth, the yield increased up to $+69.23 \%$ compared with water treatment of seeds. In the case of treatment of the root system of tomatoes with a solution of phytohormones A. calcoaceticus IMV B-7241, the number of fruits exceeded the control by 2.4 times and the total weight by $145.0 \%$.

However, it should be noted that the concentration of extracellular phytohormones synthesized by the strain was low, which significantly reduces the efficiency of the use of such preparations in plant production.

In our published review [4], we focused on the fact that most soil microorganisms, both associated and non-associated with plants, synthesize phytohormones of the auxin nature in the presence of exogenous tryptophan in the culture medium, which is the precursor of indol3 -acetic acid (IAA) synthesis. Moreover, the researchers added tryptophan into the medium at the beginning of the cultivation process and usually at a sufficiently high concentration (up to $10 \mathrm{~g} / \mathrm{L})$. We note that phytohormones are secondary metabolites, the formation of which begins in the stationary phase of growth, so it seems more logical to add a precursor at this stage of the process. In addition, the concentration of precursors used for the intensification of synthesis in microbial biotechnology, as a rule, is $0.1-0.2 \%$ of the carbon source content in the culture medium [5].

It should be noted that in [1] we found that A. calcoaceticus IMV B-7241 synthesizes auxins under growth conditions on medium with different substrates without a precursor, and therefore there are potential opportunities for enhancing its synthesis.

In connection with the above, the aim of this work is to determine the optimal concentration of tryptophan and the time of its addition into the culture medium of the surfactant producer A. calcoaceticus IMV B-7241 to ensure maximum auxin synthesis.

\section{Materials and methods}

\section{Object of research}

The object of research is Acinetobacter calcoaceticus K-4 strain, registered in Microorganisms Depositary of D.K. Zabolotny Institute of Microbiology and Virology, the National Academy of Sciences of Ukraine under the number IMV B-7241.

\section{Medium composition and conditions of cultivation}

Strain A. calcoaceticus IMV B-7241 was cultivated in the liquid medium ( $\mathrm{g} / \mathrm{L}$ distilled water): $\left(\mathrm{NH}_{2}\right)_{2} \mathrm{CO}-0.35, \mathrm{MgSO}_{4} \cdot 7 \mathrm{H}_{2} \mathrm{O}-0.1, \mathrm{NaCl}-1.0, \mathrm{Na}_{2} \mathrm{HPO}_{4}-0.6, \mathrm{KH}_{2} \mathrm{PO}_{4}-0.14$, $\mathrm{pH}$ 6.8-7.0. Yeast autolysate $-0.5 \% \mathrm{v} / \mathrm{v}$ and solution of trace elements $-0.1 \% \mathrm{v} / \mathrm{v}$ were also 
added to the medium. Trace elements solution contained (g/100 mL): $\mathrm{ZnSO}_{4} \cdot 7 \mathrm{H}_{2} \mathrm{O}-1.1$, $\mathrm{MnSO}_{4} \cdot \mathrm{H}_{2} \mathrm{O}-0.6, \mathrm{FeSO}_{4} \cdot 7 \mathrm{H}_{2} \mathrm{O}-0.1, \mathrm{CuSO}_{4} \cdot 5 \mathrm{H}_{2} \mathrm{O}-0.004, \mathrm{CoSO}_{4} \cdot 7 \mathrm{H}_{2} \mathrm{O}-0.03, \mathrm{H}_{3} \mathrm{BO}_{3}-$ $0.006, \mathrm{KI}-0.0001$, EDTA -0.5 . Crude glycerol (Komsomolsk biofuel plant, Poltava region, Ukraine) and ethanol were used as the carbon and energy sources in concentration of $2.0 \% \mathrm{v} / \mathrm{v}$.

Tryptophan was added into the medium as a $1 \%$ solution in an amount of 100, 200 or $300 \mathrm{mg} / \mathrm{L}$ at the beginning of the process or at the end of the exponential growth phase $(48 \mathrm{~h}$ of cultivation).

The culture in the exponential phase was used as the inoculum and added in concentration of $5-10 \%$ of nutritive medium volume. The concentration of the corresponding carbon source in the medium for the inoculum obtainment was $1.0 \% \mathrm{v} / \mathrm{v}$. The cultivation was carried out in $750 \mathrm{~mL}$ flasks, containing $100 \mathrm{~mL}$ of medium, on the shaker (320 rpm) at 28$30^{\circ} \mathrm{C}$ during 7 days.

\section{Obtaining of auxin extracts}

After cultivation of the strain A. calcoaceticus IMV B-7241, the biomass was separated by centrifugation $(5000 \mathrm{~g})$ for $25 \mathrm{~min}$. Residuals of sunflower oil were extracted from the cultural liquid using petroleum ether (ratio 1:1).

The extracellular phytohormones auxins were isolated by the method of redistribution of phytohormones in two immiscible phases of solvents [6]. Ethyl acetate, pH 3.0 was used as the organic solvent. The obtained extracts were evaporated under vacuum at $40-45^{\circ} \mathrm{C}$. The dry residue was redissolved in $80 \%$ ethanol and transferred into microtubes. The obtained extracts were stored at $-24^{\circ} \mathrm{C}$.

\section{Qualitative and quantitative determination of auxins}

Purification and concentration of phytohormone extracts were carried out on silicagel plates of the mark Silufol UV254 (Chemapol, Czech Republic) in a mixture of solvents used sequentially: chloroform, $12.5 \%$ aqueous ammonia, ethyl acetate:acetic acid (20:1).

The qualitative and quantitative composition of auxins was analyzed by high performance liquid chromatography (HPLC), using an Agilent 1200 liquid chromatograph (Agilent Technologies, USA) and an Agilent G1956B mass spectrometry (MS) detector. HPLC/MS analysis of auxin extracts of A. calcoaceticus IMV B-7241 was performed at the Center for Collective Use at the D.K. Zabolotny Institute of Microbiology and Virology of the NAS of Ukraine.

Standard synthetic phytohormones Sigma (Germany) and Acros Organic (Belgium) were used for comparison:

IAA - Indole-3-acetic acid;

ICal - Indole-3-carboxaldehyde;

IC - Indole-3-carbinol;

ICA - Indole-3-carboxylic acid;

IAA-hydr. - Indole-3-acetic acid hydrazide;

IBut - Indole-3-butyric acid;

Methanol (A) and 1\% acetic acid solution in water (B) were used as the mobile phase. Separation was performed on a Zorbax SB-C18 chromatographic column $(2.1 \mathrm{~mm} \times 150$ $\mathrm{mm}, 3 \mu \mathrm{m}$ ) (Agilent Technologies, USA), column flow rate $0.25 \mathrm{ml} / \mathrm{min}$, thermostat temperature $30^{\circ} \mathrm{C}$, injection volume $2 \mu$ l. Elution was performed in gradient mode: $0 \mathrm{~min}-$ $\mathrm{A}(30 \%): \mathrm{B}(70 \%) ; 25 \mathrm{~min}-\mathrm{A}(30 \%): \mathrm{B}(70 \%) ; 35 \mathrm{~min}-\mathrm{A}(100 \%): \mathrm{B}(0 \%) ; 35 \mathrm{~min}-\mathrm{A}$ $(100 \%)$ : B $(0 \%)$. 
Compound detection was performed using a diode array detector with signal recording at 254 and $280 \mathrm{~nm}$ and fixation of absorption spectra in the 191-700 nm range. Agilent G1956B mass spectrometric detector (Agilent Technologies, USA) was used to determine the molecular weights of the tested compounds. Ionization was performed in ESI and APCI mode with positive ion fixation in SCAN mode in the range of $100-1200 \mathrm{~m} / \mathrm{z}$. The calibration was performed using standard auxin solutions.

\section{Enzymatic analyzes}

Preparation of acellular extracts. To obtain acellular extracts, the culture broth obtained after cultivation of A. calcoaceticus IMV B-7241 in a liquid mineral medium with crude glycerol was centrifuged $\left(4000 \mathrm{~g}, 15 \mathrm{~min}, 4^{\circ} \mathrm{C}\right)$. The cell pellet was washed twice from the residual medium with $0.05 \mathrm{M} \mathrm{K}+$ phosphate buffer $(\mathrm{pH} 7.0)$ while centrifuging (4000 g, $15 \mathrm{~min}, 4^{\circ} \mathrm{C}$ ). The washed cells were resuspended in $0.05 \mathrm{M} \mathrm{K}+$ phosphate buffer $(\mathrm{pH} 7.0)$ and destroyed by ultrasound $(22 \mathrm{kHz}) 3$ times for $60 \mathrm{~s}$ at $4{ }^{\circ} \mathrm{C}$ on an UZDN-1 apparatus. The disintegrated cells were centrifuged $\left(12000 \mathrm{~g}, 30 \mathrm{~min}, 4{ }^{\circ} \mathrm{C}\right)$, the precipitate was discarded and the supernatant was used as an acellular extract.

Analysis of tryptophan transaminase activity. The activity of tryptophan transaminase (EC 2.6.1.27, other names: L-phenylalanine-2-oxoglutarate aminotransferase; tryptophan aminotransferase; 5-hydroxytryptophan-ketoglutaric transaminase; hydroxytryptophan aminotransferase; tryptophan aminotransferase; L-tryptophan transaminase) was determined by the formation of indole-3-pyruvate from L-tryptophan and 2-oxoglutarate, which was analyzed spectrophotometrically at $330 \mathrm{~nm}$ [7].

\section{Statistical analysis}

All the experiments were repeated three times, and the number of parallel measurements in each experiment made up 3-5. The statistical processing of the experimental data was carried out in accordance with the algorithm described in [1]. Differences of mean indicators were deemed as reliable at the significance level $\mathrm{p}<0.05$.

\section{Results and discussion}

Previous studies have shown that the synthesis of auxin metabolites was dependent on the nature of the carbon source in the culture medium of A. calcoaceticus IMV B-7241 [1].

In this work, the choice of substrates (ethanol and crude glycerol) for the cultivation of A. calcoaceticus IMV B-7241 with the aim of intensifying auxin synthesis was due to the following reasons. First, in conditions of growth on biodiesel production waste, the strain $A$. calcoaceticus IMV B-7241 synthesized the highest amount of auxins $(122.0 \mu \mathrm{g} / \mathrm{L})$ compared to that on other substrates [1]. Secondly, there is the problem of crude glycerol disposal due to the increase of biodiesel production in the world. $10 \mathrm{~kg}$ of crude glycerol is formed from every $100 \mathrm{~kg}$ of biodiesel [8]. Third, the complex microbial preparation should be characterized by high antimicrobial activity against phytopathogenic bacteria, and previously [9] it was found that such properties are inherent to the surfactants synthesized during the cultivation of A. calcoaceticus IMV B-7241 on ethanol. The intensification of IAA synthesis in the presence of tryptophan is due to the fact that in microorganisms this amino acid is a precursor of 3-indolylacetic acid biosynthesis [10]. 


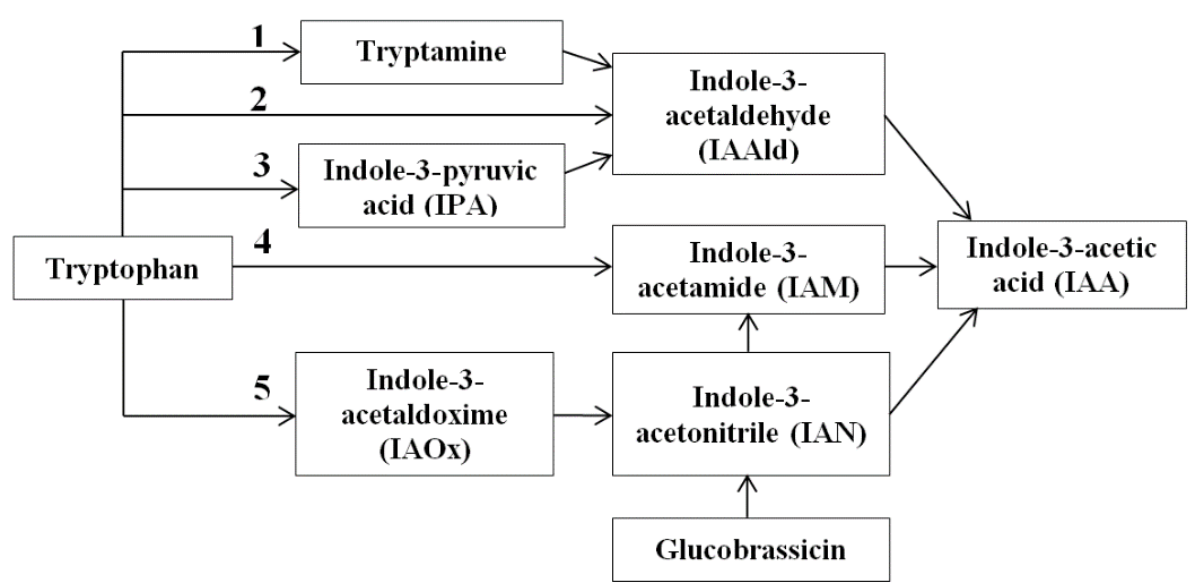

Figure 1. Ways of synthesis of indolyl-3-acetic acid from tryptophan in bacteria: 1 - through tryptamine; 2 - bypass tryptophan pathway; 3 - through indole-3-pyruvate; 4 - through indole-3-acetamide; 5 - through indole-3-acetonitrile.

Conversion of tryptophan to IAA can be accomplished in three ways (Fig. 1):

- Synthesis through indole-3-pyruvic acid and indole-3-acetic aldehyde. This is the main pathway characteristic of fungi and bacteria;

- Conversion of tryptophan to indole-3-acetic aldehyde may involve an alternative synthesis pathway in which tryptamine is formed. This pathway is found in mycorrhizal fungi and cyanobacteria.

- IAA formation via indole-3-acetamide. This is characteristic of phytopathogenic bacteria and fungi.

The data presented in the Table 1, indicate that regardless of the time of introduction of tryptophan in the culture medium of strain IMV B-7241 with crude glycerol a significant increase in the auxin synthesis compared with the indicators obtained on the medium without this precursor was observed. Among the auxins indole-3-acetic acid, indole-3-carboxylic acid, indole-3-butyric acid, indole-3-acetic acid hydrazide were identified, but the highest content was IAA, whose precursor is tryptophan.

A significant increase in auxin concentration during cultivation of A. calcoaceticus IMV B-7241 on ethanol (Table 2) was observed only when the maximum amount of tryptophan $(300 \mathrm{mg} / \mathrm{l})$ was introduced. With the introduction of $100 \mathrm{mg} / \mathrm{L}$ tryptophan, the level of auxin synthesis was virtually indistinguishable from that obtained during cultivation without the precursor. And with the addition of $200 \mathrm{mg} / \mathrm{L}$, in particular, at the end of the exponential growth phase, there was a slight increase (in 1.6 times).

It is known [5] that most precursors are involved in the processes of secondary metabolite biosynthesis at the end of exponential or early stationary growth phases. This was clearly observed under the cultivation of A. calcoaceticus IMV B-7241 on biodiesel production waste: the introduction of 100,200 , and $300 \mathrm{mg} / \mathrm{L}$ tryptophan at the end of the exponential growth phase was accompanied by an increase in the concentration of synthesized auxins in 8, 7.4, and 27.7 times, respectively (see Table 1). At the same time, other patterns were observed during the cultivation of A. calcoaceticus IMV B-7241 on ethanol medium: for most variants, the highest auxin concentration was observed when 
tryptophan was added at the beginning of the cultivation process (Table 2). Our further research will be devoted to clarifying these issues. However, it should be noted that from the point of view of the organization of technological production introduction of the precursor at the beginning of the process is much easier.

Table 1

Effect of tryptophan on the auxin synthesis under cultivation of A. calcoaceticus IMV B-7241 on raw glycerol

\begin{tabular}{|c|c|c|c|c|c|c|}
\hline \multirow{2}{*}{$\begin{array}{l}\text { The amount of } \\
\text { tryptophan, } \\
\text { mglL }\end{array}$} & \multirow[b]{2}{*}{ Growth phase } & \multicolumn{4}{|c|}{ The concentration of auxins, $\mu \mathrm{g} / \mathrm{L}$} & \multirow{2}{*}{$\begin{array}{c}\text { The amount } \\
\text { of auxins, } \\
\mu \mathrm{g} / \mathrm{L}\end{array}$} \\
\hline & & IAA & ICA & IBut & $\begin{array}{l}\text { IAA- } \\
\text { hydr }\end{array}$ & \\
\hline 0 & Lag phase & 150.8 & 24.6 & - & - & 175.4 \\
\hline \multirow[b]{2}{*}{100} & Lag phase & 207.65 & 171.15 & - & - & 378.80 \\
\hline & $\begin{array}{l}\text { The end of the } \\
\text { exponential } \\
\text { phase }\end{array}$ & 359.85 & 309.22 & - & 735.66 & 1404.73 \\
\hline \multirow[b]{2}{*}{200} & Lag phase & 242.46 & 184.38 & - & - & 426.84 \\
\hline & $\begin{array}{l}\text { The end of the } \\
\text { exponential } \\
\text { phase }\end{array}$ & 694.74 & 600.3 & - & - & 1295.04 \\
\hline \multirow[b]{2}{*}{300} & Lag phase & 1123.0 & 401.70 & 47.74 & - & 1572.44 \\
\hline & $\begin{array}{l}\text { The end of the } \\
\text { exponential } \\
\text { phase }\end{array}$ & 4091.0 & 717.89 & 42.09 & - & 4850.98 \\
\hline
\end{tabular}

Note. IAA - Indole-3-acetic acid; ICA - Indole-3-carboxylic acid; IBut - Indole-3-butyric acid; IAA-hydr - Indole-3-acetic acid hydrazide. «-»- not found.

Table 2

Synthesis of auxins under cultivation of $A$. calcoaceticus IMV B-7241 on tryptophan-containing ethanol medium

\begin{tabular}{|c|c|c|c|c|}
\hline \multirow{2}{*}{$\begin{array}{l}\text { The amount of } \\
\text { tryptophan, mg\L }\end{array}$} & \multirow[t]{2}{*}{ Growth phase } & \multicolumn{2}{|c|}{$\begin{array}{c}\text { The concentration of } \\
\text { auxins, } \mu \mathrm{g} / \mathrm{L}\end{array}$} & \multirow{2}{*}{$\begin{array}{c}\text { The amount } \\
\text { of auxins, } \\
\mu \mathrm{g} / \mathrm{L}\end{array}$} \\
\hline & & IAA & ICA & \\
\hline 0 & Lag phase & 173.32 & 47.10 & 220.42 \\
\hline \multirow[b]{2}{*}{100} & Lag phase & 126.98 & 113.89 & 240.87 \\
\hline & $\begin{array}{l}\text { The end of the } \\
\text { exponential phase }\end{array}$ & 130.08 & 95.67 & 225.75 \\
\hline \multirow[b]{2}{*}{200} & Lag phase & 135.67 & 97.30 & 232.97 \\
\hline & $\begin{array}{l}\text { The end of the } \\
\text { exponential phase }\end{array}$ & 136.04 & 216.84 & 352.88 \\
\hline \multirow{2}{*}{300} & Lag phase & 995.47 & 1242.0 & 2261.66 \\
\hline & $\begin{array}{l}\text { The end of the } \\
\text { exponential phase }\end{array}$ & 710.13 & 396.74 & 1106.87 \\
\hline
\end{tabular}

Note. IAA - Indole-3-acetic acid; ICA - Indole-3-carboxylic acid. 
The data presented in the Tables 1 and 2 indicate that the concentration of synthesized auxins increased with increasing of precursor concentration in the culture medium of $A$. calcoaceticus IMV B-7241. It is possible that further increase of tryptophan will be accompanied by intensification of auxin synthesis. However, at this stage, for the creation of an effective microbial preparation with growth-stimulating properties it is unnecessary, because with the achieved auxin concentration $(2000-5000 \mu \mathrm{g} / \mathrm{L}$, see Tables 1 and 2) the culture broth of A. calcoaceticus IMV B-7241 with the purpose of seed or roots treatment of plants seedlings must be diluted at least in 400-500 times.

To confirm that exogenous tryptophan is involved in auxin biosynthesis, the activity of one of the key enzymes for the synthesis of indole-3-acetic acid (tryptophan transaminase) was analyzed. Tryptophan transaminase catalyzes the reaction of the formation of indole-3pyruvic acid from L-tryptophan and 2-oxoglutarate. According to the data shown in Figure 2, when cultivating A. calcoaceticus IMV B-7241 on the crude glycerol medium with 300 $\mathrm{mg} / \mathrm{L}$ tryptophan the activity of this enzyme was higher than on the medium without this precursor. In addition, it should be noted that when introducing tryptophan at the end of the exponential growth phase, the activity of tryptophan transaminase was in 3.2 times higher than when tryptophan was introduced in the lag phase, which is consistent with the data given in Table 1 regarding the concentration of formed auxins. These data suggest that IAA biosynthesis in A. calcoaceticus IMV B-7241 going through the formation of indole-3pyruvate (see Figure 1).

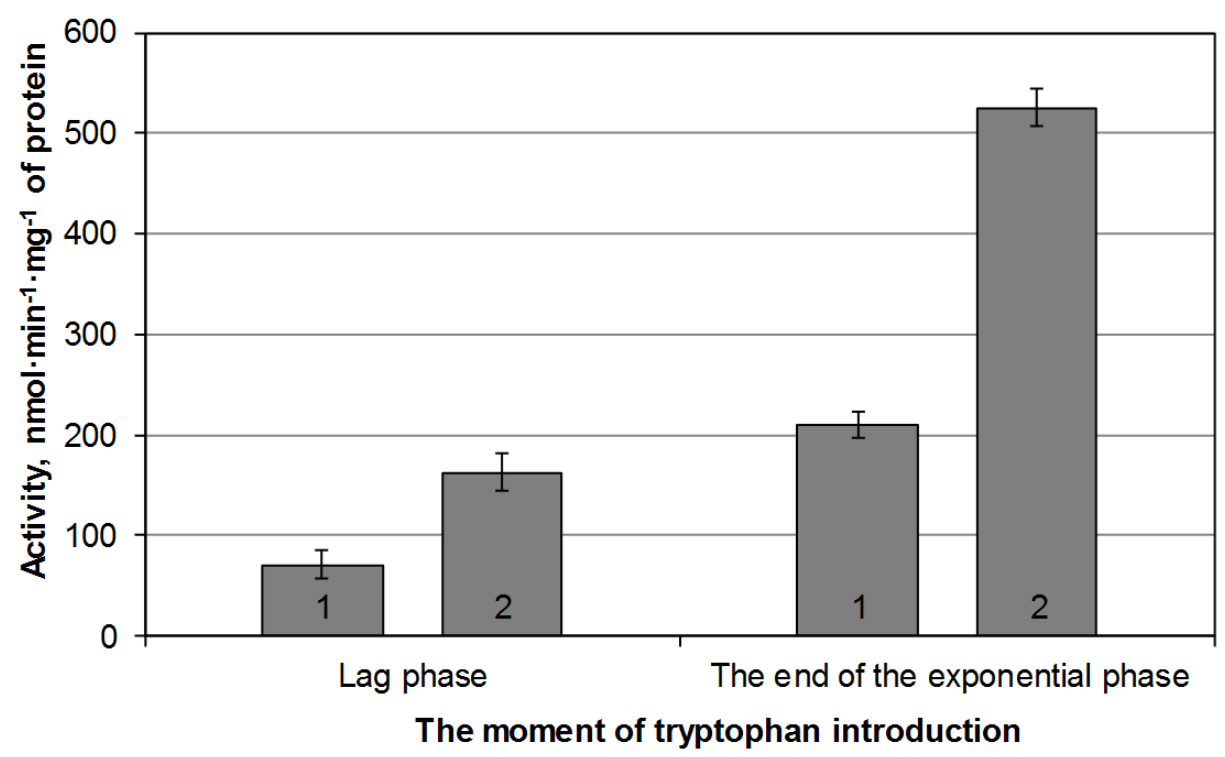

Figure 2. The effect of tryptophan on the activity of tryptophan transaminase under different conditions of cultivation of $A$. calcoaceticus IMV B-7241

1 - without tryptophan; 2 - tryptophan, $300 \mathrm{mg} / \mathrm{L}$ 
In 2015-2016, we published two papers [12,13] in which we first reported the ability of surfactant producers Rhodococcus erythropolis IMV Ac-5017, A. calcoaceticus IMV B7241 and Nocardia vaccinii IMV B-7405 to synthesize phytohormones of auxin nature. Only after the publication of this work it was reported that indolyl-3-acetic acid was formed by bacteria (mainly Rhodococcus species) isolated from contaminated hydrocarbons and heavy metals [60]. However, the authors have established the ability to synthesize surfactants by the index of emulsification and the reduction of surface tension, which appeared to be insignificant - up to $60-65 \mathrm{mN} / \mathrm{m}$ (against $30-35 \mathrm{mN} / \mathrm{m}$ in the surfactant producers).

In 2018, papers [14-16] were published in which the ability of producers of surfaceactive lipopeptides and rhamnolipids to synthesize phytohormones of auxin nature was established. Thus, endophytic strain Bacillus sp. Fcl1 [14] synthesized iturin A and surfactin, which had antimicrobial effects on the phytopathogenic fungi of the genera Fusarium, Phytophthora, Sclerotium, Corynespora, as well as indole-3-acetic acid, presence of which in the culture broth was determined by a qualitative reaction with a Salkowski reagent. The authors did not analyze the concentration of synthesized lipopeptides and indolyl-3-acetic acid.

Bacillus sp. B19, Bacillus sp. P12 and B. amyloliquefaciens B14, isolated from the soil, synthesize a complex of antimicrobial compounds (surface-active lipopeptides kurstakin, surfactin, iturin, fengycin and antibiotic polymyxin), as well as auxins [15]. The concentration of auxins synthesized by strains B19 and P12 was 5.71 and $4.90 \mathrm{mg} / \mathrm{L}$, respectively. Tryptophan was not added to the culture medium. However, tryptone which contains tryptophan was used as a carbon source for cultivation of producers.

The endophytic strain of Pseudomonas aeruginosa L10 [16] under the cultivation on diesel fuel (5 g/l) synthesized rhamnolipids, which reduced the surface tension to $29.5 \mathrm{mN} / \mathrm{m}$, and indolyl-3-acetic acid at a concentration of $27 \mu \mathrm{g} / 1$. It should be noted that in this work the authors did not try to increase the synthesis of IAA.

It is worth noting that after the publication of our review [4], in which the existing literature on the synthesis of phytohormones by different microorganisms was analyzed, there were papers in which the researchers compared the synthesis of auxins in the absence and presence of precursors.

The causative agent of tomato disease $P$. syringae DC3000 synthesizes IAA through the formation of indole-3-acetaldehyde from indole-3-pyruvate (see Fig. 2) [17]. When 0.25 $\mathrm{mM}$ tryptophan was added to the culture medium, the IAA concentration was $2.7 \mu \mathrm{g} / \mathrm{ml}$, which is several orders of magnitude higher than without the introduction of the biosynthesis precursor $(0.03 \mathrm{mg} / \mathrm{L})$. At the same time, the researchers tested the effects of other precursors on the formation of IAA, in particular, the effects of indole-3-acetaldehyde and indole-3acetonitrile. Thus, with introduction into the culture medium of these precursors, the concentration of auxin at $48 \mathrm{~h}$ of cultivation was $11.7 \mu \mathrm{g} / \mathrm{ml}$ and $14.1 \mu \mathrm{g} / \mathrm{ml}$, respectively.

Analysis of the genome and metabolic pathways of Variovorax boronicumulans strain CGMCC 4969 shows that IAA biosynthesis in these bacteria begins with indole-3acetonitrile, which enters the cell exogenously. Therefore, with addition to the culture medium of acetonitrile and cobalt, the concentration of phytohormone after 60 hours of cultivation was $1.88 \mathrm{mmol} / \mathrm{L}$ (the concentration of IAA synthesized without a precursor was not determined).

Scientists from Thailand and Japan [18] have established the ability of the endophytic fungi Colletotrichum fructicola CMU-A109 to synthesize IAA in high concentrations in the presence of tryptophan. After 26 days of CMU-A109 cultivation, the IAA concentration was $1.2 \mathrm{mg} / \mathrm{L}$. 
When introducing tryptophan into the culture medium of the strains of Mortierella sp. MA DEM7 and MA DEM32 [19], an intensification of IAA synthesis was observed. The highest concentration $(32 \mathrm{mg} / \mathrm{L})$ was reached after 9 days of cultivation of the MA DEM32 strain on the medium with $1.5 \mathrm{mM}$ tryptophan. In the case of growing of MA DEM7 strain in the presence of $3.0 \mathrm{mM}$ tryptophan, the amount of synthesized IAA was twice lower.

Thus, analysis of the literature has shown that introduction of biosynthesis precursors is effective for enhancing the synthesis of phytohormones. However, the authors of works [17-19] added tryptophan in fairly high concentrations. The use of large quantities of tryptophan as a component of the nutrient medium is not economically feasible. Our studies have shown that at significantly lower concentrations of tryptophan, the intensification of the auxin synthesis is in hundreds of times.

In addition, most scientists are studying the synthesis of phytohormones on rich nutrient medium that contain glucose, sucrose, dextrose, glucuronic acid, peptone, tryptone, mannitol as carbon source $[10,11,15,16]$. Such mediums for growing phytohormone producers are expensive, so there is a need to reduce their cost, in particular by finding cheaper carbon substrates. We have for the first time established the possibility of the auxin formation on a cheap medium using waste biodiesel production as a substrate. There is no such information in the literature.

\section{Conclusion}

Thus, the result of the work established the possibility of increasing by one or two orders of magnitude of the synthesized auxins in the case of introduction low concentrations of their biosynthesis precursor in the culture medium of strain A. calcoaceticus IMV B-7241 with biodiesel production waste. The obtained results are the basis for increasing the efficiency of the use of the complex preparation in plant production.

\section{References}

1. Pirog T.P., Leonova N.O., Shevchuk T.A., Havrylkina D.V. (2017), Influence of cultivation conditions of surfactants producers Acinetobacter calcoaceticus IMV B-7241, Rhodococcus erythropolis IMV Ac-5017 and Nocardia vaccinii IMV B-7405 on phytogormones synthesis, Scientific works of NUFT, 23(5), pp. 15-22, DOI: 10.24263/2225-2924-2017-235-1-4.

2. Havrylkina D.V., Leonova N.O., Pirog T.P. (2019), The influence of exometabolites Nocardia vaccinii IMV B-7405, Acinetobacter calcoaceticus IMV B-7241 and Rhodococcus erythropolis IMV Ac-5017 on yields of tomatoes and barley, Journal of Agriculture and Environment, 1(9), pp. 1-8, DOI: 10.23649/jae.2019.1.9.2.

3. Piatetska D.V., Leonova N.O., Pirog T.P., Klymenko N.O. (2019), Yield of tomatoes and peppers under the influence of Nocardia vaccinii IMV B-7405, Acinetobacter calcoaceticus IMV B-7241 and Rhodococcus erythropolis IMV Ac-5017, Journal of Agriculture and Environment, 3(11), pp. 1-8, DOI: 10.23649/jae.2019.3.11.4.

4. Pirog T.P., Iutynska G.O., Leonova N.O., Beregova K.A., Shevchuk T.A. (2018), Microbial synthesis of phytohormones, Biotechnologia Acta,11(1), pp. 5-24, DOI: 10.15407/biotech11.01.005.

5. Pidgorsky V.S., Iutynska G.O., Pirog T.P. (2010), Intensification of microbial synthesis technologies, Naukova knyha, Kyiv.

6. Negretsky V.A. (1988), Guidelines for the determination of phytohormones, Institute of 
Botany, Academy of Sciences of the Ukrainian SSR, Kiev. (In Russian).

7. Collier R.H., Kohlhaw G. (1972), Nonidentity of the aspartate and the aromatic aminotransferase components of transaminase A in Escherichia coli, Journal of Bacteriology, 112(1), pp. 365-71.

8. Zhou J. J., Shen J. T., Jiang L. L., Sun Y. Q., Mu Y., Xiu Z. L. (2017), Selection and characterization of an anaerobic microbial consortium with high adaptation to crude glycerol for 1,3-propanediol production, Applied Microbiology and Biotechnology, 101, pp. 59855996, DOI: $10.1007 / \mathrm{s} 00253-017-8311-8$

9. Pirog T.P., Savenko I.V., Shevchuk T.A. (2016), Antimicrobial properties of surfactants synthesized under different cultivation conditions of Acinetobacter calcoaceticus IMV B7241, Mikrobiologichny Zhurnal, 78(3), pp. 2-12.

10. Gopalakrishnan S., Sathya A., Vijayabharathi R., Varshney R.K., Gowda C.L., Krishnamurthy L. (2015), Plant growth promoting rhizobia: challenges and opportunities, 3 Biotech, 5(4), pp. 355-377, DOI: 10.1007/s13205-014-0241-x.

11. Shao J., Li S., Zhang N., Cui X., Zhou X., Zhang G., Shen Q., Zhang R. (2015), Analysis and cloning of the synthetic pathway of the phytohormone indole-3-acetic acid in the plantbeneficial Bacillus amyloliquefaciens SQR9, Microial Cell Factories, 14, pp. 130, DOI: 10.1186/s12934-015-0323-4

12. Pirog T.P., Leonova N.O., Shevchuk T.A., Savenko I.V., Iutinska H.O. (2016), Synthesis of phytohormones bacteria of Acinetobacter calcoaceticus IMV B-7241, Rhodococcus erythropolis IMV Ac-5017 and Nocardia vaccinii IMV B-7405 - producers of surfaceactive substances, Proceedings of the National Academy of Sciences of Belarus. Biological series, 1, pp. 90-95.

13. Pirog T.P., Leonova N.O., Shevchuk T.A., Panasuk E.V., Beregovaya K.A., Iutynskaya G.A. (2015), Synthesis of phytohormones by Nocardia vaccinii IMV B-7405 - producer of surfactants, Mikrobiologichny Zhurnal, 77(6), pp. 21-30.

14. Jayakumar A., Krishna A., Mohan M., Nair I.C., Radhakrishnan E.K. (2018), Plant growth enhancement, disease resistance, and elemental modulatory effects of plant probiotic endophytic Bacillus sp. Fcl1, Probiotics and Antimicrobial Proteins, 11(2), pp. 526-534, DOI: $10.1007 / \mathrm{s} 12602-018-9417-8$.

15. Sabaté D.C., Brandan C.P., Petroselli G., Erra-Balsells R., Audisio M.C. (2018), Biocontrol of Sclerotinia sclerotiorum (Lib.) de Bary on common bean by native lipopeptide-producer Bacillus strains, Microbiology Results, 211, pp. 1-30, DOI: 10.1016/j.micres.2018.04.003

16. Wu T., Xu J., Xie W., Yao Z., Yang H., Sun C. (2018), Pseudomonas aeruginosa L10: a hydrocarbon-degrading, biosurfactant-producing and plant-growth-promotion endophytic bacterium isolated from a reed (Phragmites australis), Front Microbiology, 9, pp. 1087, DOI: $10.3389 /$ fmicb.2018.01087.

17. McClerklin S., Goo Lee S., Harper C. P., Nwumeh R., Jez M., Kunkel B. N. (2018), Indole3 -acetaldehyde dehydrogenase-dependent auxin synthesis contributes to virulence of Pseudomonas syringae strain DC3000, PLoS Pathogens, 14(1), pp. 1-24, DOI: 10.1371/journal.ppat.1006811.

18. Numponsak T., Kumla J., Suwannarach N., Matsui K., Lumyong S. (2018), Biosynthetic pathway and optimal conditions for the production of indole-3-acetic acid by an endophytic fungus, Colletotrichum fructicola CMU-A109, PLoS One, 13(10), pp. 1-17, DOI: 10.1371/journal.pone.0205070.

19. Ozimek E., Jaroszuk-Scisel J., Bohacz J., Kornillowicz-Kowalska T., Tyskiewicz R., Slomka A., Nowak A., Hanaka A. (2018), Synthesis of indoleacetic acid, gibberellic acid and ACC-deaminase by Mortierella strains promote winter wheat seedlings growth under different conditions, International Journal of Molecular Sciences, 19(10), pp. 1-17, DOI: 10.3390/ijms19103218. 